\title{
Overexpression of SHP2 tyrosine phosphatase promotes the tumorigenesis of breast carcinoma
}

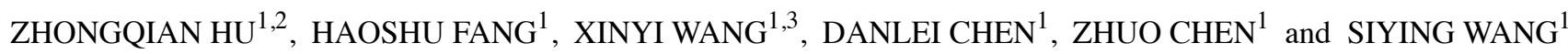 \\ ${ }^{1}$ Department of Pathophysiology, Anhui Medical University, Hefei, Anhui 230032; \\ ${ }^{2}$ Department of Ultrasound, Zhongda Hospital, Southeast University, Nanjing, Jiangsu 210009; \\ ${ }^{3}$ Department of Clinical Medicine, Anhui Medical University, Hefei, Anhui 230032, P.R. China
}

Received March 13, 2014; Accepted April 30, 2014

DOI: $10.3892 /$ or.2014.3201

\begin{abstract}
Expression of Src homology phosphotyrosine phosphatase 2 (SHP2) has been observed in human breast cancer. SHP2 is known to promote cell migration and invasiveness. However, the pathophysiologic role of SHP2 and its relevance to tumorigenesis are still largely unknown. In the present study, we aimed to evaluate the effect of SHP2 on the malignant phenotype of human breast cancer. An SHP2-overexpressing human breast cancer cell line was established by stable transfection of the SHP2 vector. The expression of SHP2 protein was detected using western blotting. The effects of SHP2 overexpression on cell proliferation were examined by an MTS assay. Invasion and migration abilities of the SHP2-overexpressing cells were determined using a Matrigel-based Boyden chamber invasion assay and a monolayer wound-healing assay. Increased SHP2 expression was detected following SHP2-vector transfection in the MDA-MB-231 cells. Overexpression of SHP2 was associated with increased cell proliferation and clone formation, and decreased chemotherapeutic sensitivity. Furthermore, transfection of SHP2 into breast cancer cells significantly promoted tumor growth in a mouse xenograft model. The mechanism of the promotion of tumorigenesis by SHP2 appears to involve its ability to increase the activity of ERK/ AKT-mediated signaling pathways. In conclusion, our data suggest an important role of SHP2 in the molecular etiology of tumor growth, and implicate the potential application of SHP2 in cancer therapy.
\end{abstract}

Correspondence to: Dr Siying Wang, Department of Pathophysiology, Anhui Medical University, 81 Meishan Road, Hefei, Anhui 230032, P.R. China

E-mail: sywang@ahmu.edu.cn

Abbreviations: SHP2, Src homology phosphotyrosine phosphatase 2

Key words: breast cancer, SHP2, overexpression

\section{Introduction}

Cytokine, growth factor and integrin signaling are regulated by $\mathrm{SH} 2$ domian-containing tyrosine phosphatase 2 (SHP2). SHP2 has two SH2 domains within the N-terminus part, and a phosphatase domain within the C-terminal part $(1,2)$. SHP2 is expressed ubiquitously in mammalian tissue $(3,4)$, and regulates several cellular processes $(5,6)$. In general, SHP2 stimulates the release of cytokines and growth factors, i.e. insulin, EGF, PDGF, FGF, IL-1 and IL-6 (7,4). Activation of SHP2 may interfere with MAPK cellular signal transduction via Erk, JNK/SAPK, p38/RK and BMK1/Erk5 (8). Furthermore, SHP2 is a bona fide oncogene, as overexpression of SHP2 has been frequently observed in adult human leukemia $(9,10)$. Genetic analyses revealed that germline mutations of PTPN11, which encodes SHP2 in humans, present in nearly $50 \%$ of patients with developmental abnormalities, such as leukemia and solid tumors (11-16).

Overexpression of SHP2 has been observed in most breast cancer cell lines and in breast cancer tissues $(8,17)$, and is accompanied by lymph node metastasis. Inhibition of SHP2 in breast cancer cell lines was found to abolish the growth and decrease the survival of tumor cells, leading to the differentiation of malignant cells into a normal breast epithelial phenotype $(12,18,19)$. These observations suggest that SHP2 promotes tumor development through increased tumor formation and metastasis.

However, the mechanism involved in the promotion of the malignant potential of breast cancer by SHP2 is largely unknown. To determine the influence of the SHP-2 signaling pathway on the developmental process of breast cancer, we constructed an SHP2 eukaryotic expression vector and transfected it into MDA-MB-231 cells. Our results clearly showed that transfection of SHP2 into MDA-MB-231 cells resulted in an altered phenotype. SHP2 overexpression was associated with significantly increased cell proliferation and clone formation, and decreased chemotherapeutic sensitivity in the SHP2-MB-231 group when compared with the control groups. Anchorage-independent growth, migration and invasion of the transfected cells in vitro were also increased. These findings support the hypothesis that SHP2 is a cancer agonist; overexpression of SHP2 contributes to the malignant progression of breast cancers. 


\section{Materials and methods}

Plasmids, cell culture and transfection. The SHP2 mammalian expression vector was constructed as described previously $(18,20)$. Briefly, SHP2 cDNA was obtained using RT-PCR from mouse embryonic fibroblasts, and was cloned into the pcDNA3.1 vector. MDA-MB-231 human breast cancer cells were cultured in DMEM with $10 \%$ heat-inactivated fetal bovine serum (FBS). MDA-MB-231 cells were transfected with the pcDNA3.1 empty vector or the SHP2-pcDNA3.1 vector using Lipofectamine 2000 reagent (Invitrogen, Carlsbad, CA, USA). After $24 \mathrm{~h}$, fresh DMEM was added which contained G418 $(800 \mu \mathrm{g} / \mu \mathrm{l})$. The culture medium was replaced thrice weekly until stably transfected colonies were observed.

Immunoblotting. Immunoblotting was performed as previously described (18). In brief, $15 \mu \mathrm{g}$ of the protein samples was separated on an SDS-polyacrylamide gel by electrophoresis. The separated proteins were transferred to nitrocellulose membranes. The membranes were blocked in PBST (5\% BSA, $0.05 \%$ Tween-20, $\mathrm{pH}$ 7.4) at room temperature for $1 \mathrm{~h}$. Subsequently, the membranes were probed with primary antibodies directed against target proteins overnight at $4^{\circ} \mathrm{C}$. After three washes in PBST, the membranes were incubated with the secondary antibody conjugated to horseradish peroxidase (Amersham, Arlington Heights, IL, USA). Signals were detected using a chemiluminescence method (Amersham). The same membranes were stripped with stripping buffer for $15 \mathrm{~min}$ at room temperature and immunoblotted with antiacting antibody. Digitalization of films was performed using ImageJ 1.43G (NIH, Bethesda, MD, USA).

Cell adhesion assay. MB-231, vector-MB-231 and SHP2-MB231 cells were collected upon trypsinization, and washed in serum-free DMEM ( $0.2 \%$ trypsin inhibitor). Cells were resuspended at $10^{5}$ cells $/ \mathrm{ml}$ in DMEM (10\% FBS), and cultured in fibronectin (FN) $(10 \mathrm{mg} / \mathrm{ml})$-pre-coated 96-well plates $(100 \mu \mathrm{l}$ suspension/well).

After $30 \mathrm{~min}, 1 \mathrm{~h}$ and $2 \mathrm{~h}$, non-adherent cells were removed by washing the culture plate with PBS. Attached cells were stained with $0.1 \%$ crystal violet in $20 \%$ methanol. The crystal violet staining was eluted with $0.1 \mathrm{M}$ sodium citrate $(\mathrm{pH} 4.2)$, and the plate was measured at $490 \mathrm{~nm}$ using a microplate reader.

Cell migration assay. Cell migration was analyzed using Transwell System (Costar Corp., Acton, MA, USA). MDA-MB231 cells (SHP2- or vector-transfected) were trypsinized, and $1 \times 10^{5}$ cells were added to the top chambers of the Transwell (8- $\mu \mathrm{m}$ pore size; BD Bioscience, Bedford, MA, USA), and assay medium was added to the bottom chambers and incubated at $37^{\circ} \mathrm{C}$ in $5 \% \mathrm{CO}_{2}$ for $12 \mathrm{~h}$. The membrane was fixed with methanol, and the cells remaining on the upper chamber were removed. The migrated cells were stained with Giemsa and were counted. For each well, five microscopic fields were counted randomly. Data obtained from triplicate wells were analyzed.

Wound-healing assay. Wound-healing assay was performed as previously described (21). Briefly, MB-231, vector-MB-231 and
SHP2-MB-231 cells were seeded in 6-well dishes $\left(1 \times 10^{5}\right.$ cells/ well) and starved overnight. A single scratch wound was generated using a pipette tip. The cells were cultured in DMEM with $5 \%$ FBS. After 0, 24 and $48 \mathrm{~h}$, images were captured using an inverted microscope, and the width of the wounded area was measured. The relative migration distance was calculated with the following formula: Migration distance $=[($ width at $0 \mathrm{~h}-$ width at observation point)/width at $0 \mathrm{~h}$ ] x 100\%

Cell growth assay. Anchorage-independent growth was assessed by a soft agar clonogenic assay. Cells were detached and plated in $0.6 \%$ agarose with a $1.2 \%$ agarose underlay ( $1 \times 10^{3}$ cells per well in 6 -well plates). The number of foci was counted after 18 days. For the focus formation assay, the cells were reseeded and cultured for 4 weeks in DMEM with $10 \%$ FBS. The cells were fixed with formalin and stained with $0.1 \%$ crystal violet.

Cell proliferation assay. MB-231, vector-MB-231 and SHP2MB-231 cells were plated at $1 \times 10^{4}$ cells/well in 96-well plates. After $24 \mathrm{~h}$, cisplatin $(4 \mu \mathrm{g} / \mathrm{ml})$ or DMSO was added to the culture medium. The cells were harvested at 24,48 and $72 \mathrm{~h}$ and were counted using a hemocytometer. The experiments were repeated in triplicate. The proliferation rate was calculated using the following formula: Proliferation rate $=[$ (cell number at harvest time - cell number at $0 \mathrm{~h}$ )/cell number at $0 \mathrm{~h}] \mathrm{x} 100 \%$.

In vivo tumorigenicity assay. Tumor cells ( $\left.2 \times 10^{6} / 100 \mu 1 \mathrm{PBS}\right)$ were injected subcutaneously into the mid-dorsum of BALB/c nude mice (4-6 weeks old) in a total volume of $100 \mu$ l. Mice were monitored weekly for tumor development. Tumor sizes were measured using vernier calipers, and the tumor volume was calculated according to the formula: length $\mathrm{x}$ width $^{2} \mathrm{x} 10$, which approximates the volume of an elliptical solid. Mice were sacrificed 50 days after injection, and the tumor tissues were harvested. Upon removal of the tumors, the tumor volume was calculated using the equation: Tumor volume $=\left(\right.$ length $\mathrm{x}$ width $\left.{ }^{2}\right) / 2$. Tumor samples were fixed in $10 \%$ formalin for further analysis.

Histological staining. Immunohistochemical procedure was performed as previously described (21). Tumor samples fixed in $10 \%$ neutral buffered formalin for at least $24 \mathrm{~h}$. Paraffin embedding was performed, and sections $(3-\mu \mathrm{m})$ were cut and stained with hematoxylin and eosin (H\&E).

CD31 immunohistochemistry. Sections $(4 \mu \mathrm{m})$ were blocked with $1 \%$ BSA and $0.01 \%$ TritonX-100 for $1 \mathrm{~h}$ at room temperature, and incubated with the primary antibody (anti-CD31, PECAM, 1:50) overnight at $4^{\circ} \mathrm{C}$. Negative controls were performed by either omitting the primary antibody or incubating sections with normal rat IgG. Detection was performed by incubation with biotinylated secondary antibodies (Vector Laboratories Inc., Burlingame, CA, USA) for $1 \mathrm{~h}$ at room temperature. The sections were incubated with the avidin-biotin-peroxidase complex (Vector Laboratories Inc.; 1:100 in PBS) for $1 \mathrm{~h}$ and developed in $0.05 \%$ 3,3'-diaminobenzidine (Sigma) containing $0.003 \% \mathrm{H}_{2} \mathrm{O}_{2}$ in PBS. To determine the average microvessel density (AMVD), the number of CD31-positive microvessels was counted in 10 randomly chosen visual fields under micros- 

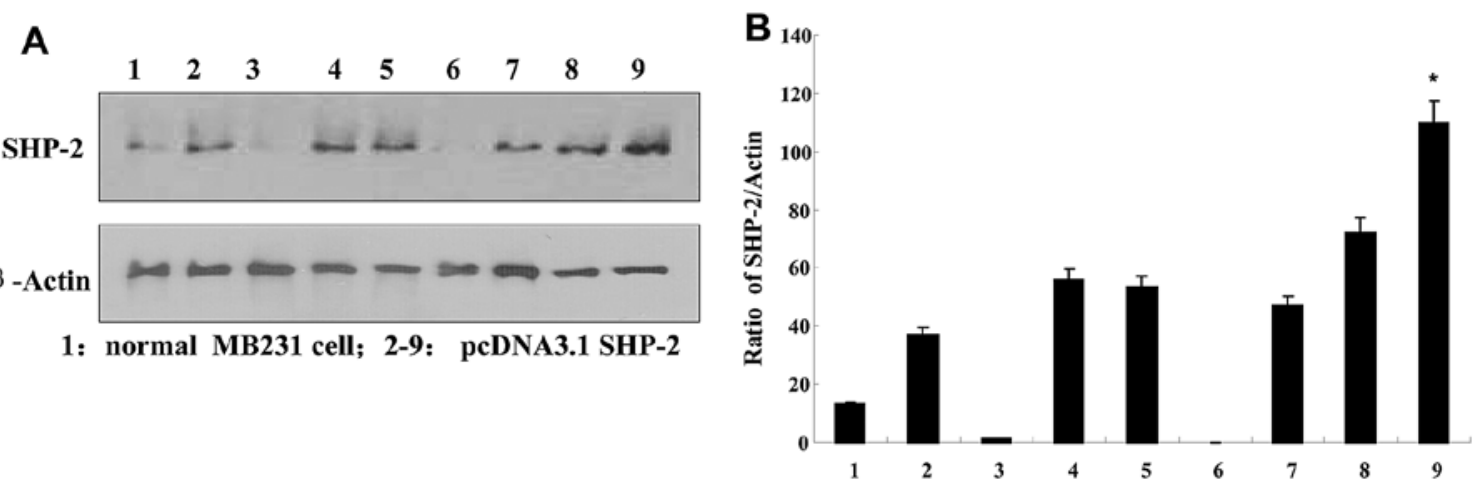

Figure 1. SHP2 protein expression in the SHP2-MB-231 cells. (A) Western blotting was performed to examine SHP2 expression after transfection of pcDNA SHP2 in the MDA-MB-231 cells. (B) SHP2 expression was normalized to the constitutively expressed $\beta$-actin ("P<0.05).

copy. The AMVD was calculated and expressed as the number of microvessels per $\mathrm{mm}^{2}$ area.

Data and statistical analyses. All experiments were repeated a minimum of 3 times. Data are shown as mean values \pm standard deviation. Groups were compared by one-way ANOVA followed by Pearson coefficient analysis for bivariate correlation. A P-value of $<0.05$ was considered to indicate a statistically significant result.

\section{Results}

SHP2 protein expression in the transfected breast cancer cells. The expression of SHP2 was examined in each of the transduced populations. After transfection with pcDNA3.1SHP2, G418-resistant MDA-MB-231 clones were expanded as monoclonal populations. Four weeks later, 15 clones were selected to examine SHP2 expression by performing western blotting. As shown in Fig. 1, high expression of SHP2 was detected in the respective clones, when compared with the non-transfected MB231 cells. Colonies of line 9 were chosen for subsequent experiment, and named SHP2-MB-231. The expression of SHP2 was normalized to the constitutively expressed $\beta$-actin protein using densitometry.

Overexpression of SHP2 is associated with the increased adhesion of MB231 cells to FN. We examined the adhesive ability of the MB-231, vector-MB-231 and SHP2-MB-231 cells to FN. As shown in Fig. 2, the number of adhesive cells in the SHP2-MB-231 group was significantly increased when compared with the MB-231 and vector-MB-231 groups (Fig. 2A). Of note, after $2 \mathrm{~h}$ of incubation, SHP2-transfected cells exhibited an extensively elongated appearance, while most cells in both control groups appeared round (Fig. 2B). Moreover, the absorbance value in the SHP2-MB-231 group was increased 3 - and 2-fold at $30 \mathrm{~min}$ and $1 \mathrm{~h}$, respectively, when compared with the control group (Fig. 2C).

Overexpression of SHP2 enhances migration and invasion of $M D A-M B-231$ cells. A wound-healing assay was performed to investigate whether SHP2 overexpression affects tumor migration in vitro. As shown in Fig. 3A, overexpression of SHP2 significantly increased the migration of MB-231 cells, as the width of the scratch was markedly decreased after $48 \mathrm{~h}$. In

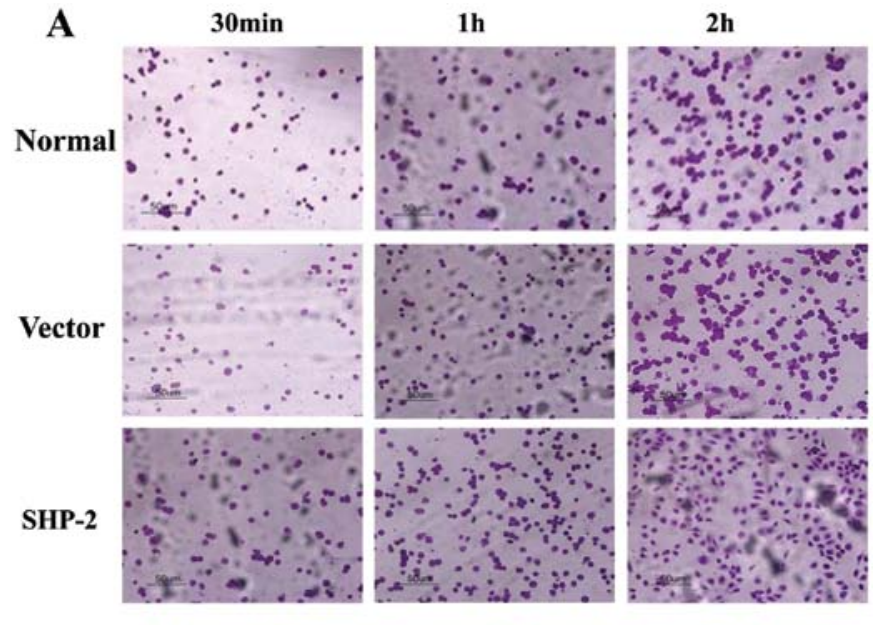

B

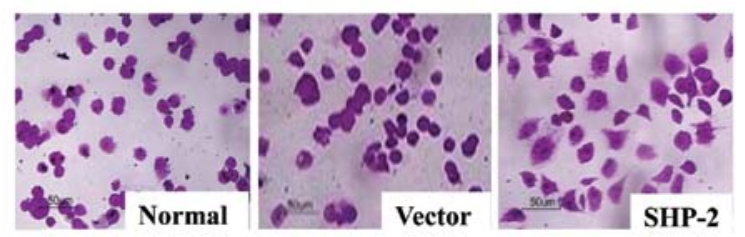

C

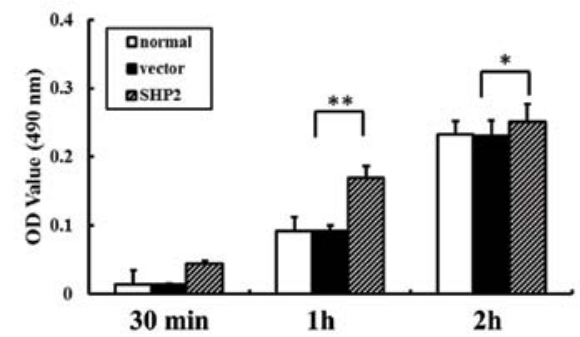

Figure 2. Overexpression of SHP2 enhances the adhesion of MDA-MB-231 cells to fibronectin (FN). (A) FN-adhesive cells were detected by performing crystal violet staining (original magnification x100). (B) Morphological evaluation of adhesive cells under a high magnification field (original magnification $\mathrm{x} 400$ ). (C) The absorbance values were compared at $490 \mathrm{~nm}$ to indicate the adhesive abilities of the different groups $\left({ }^{*} \mathrm{P}<0.05,{ }^{* *} \mathrm{P}<0.01\right)$.

contrast, the width of the scratch was only slightly decreased in the MB-231 control group, as well as the vector-MB-231 group. Moreover, overexpression of SHP2 in the SHP2-MB231 group showed a similar pattern in the transwell experiment (Fig. 3B). After $12 \mathrm{~h}$ of incubation, the invasive cells in the vector-MB-231 group was 4-fold higher when compared with the control groups (Fig. 3C). 
A Normal

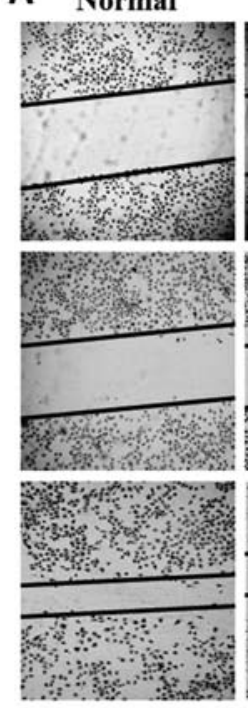

Vector
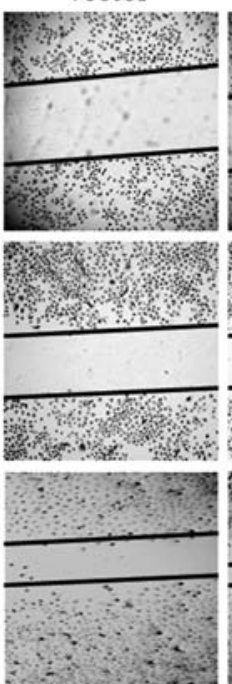

WT

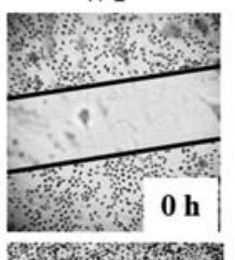

$\mathbf{0 h}$
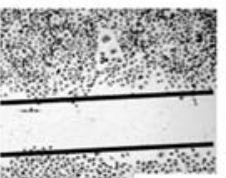

24 h

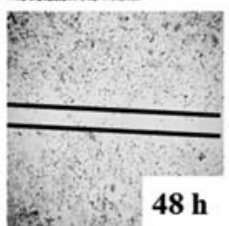

B

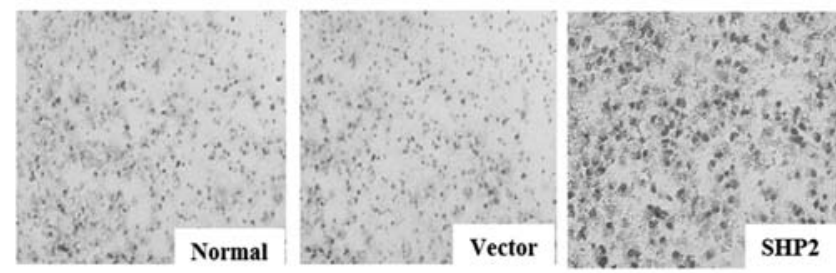

C

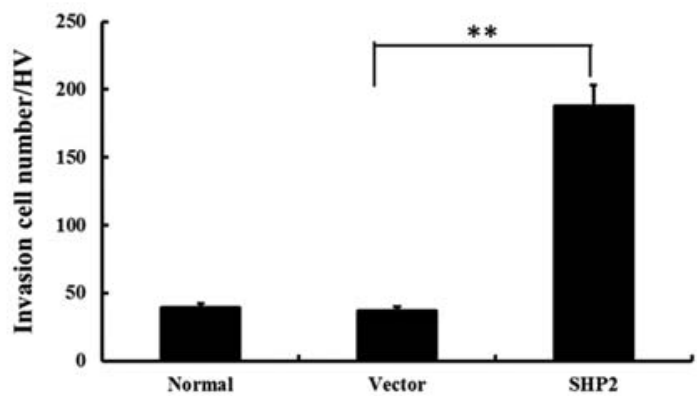

Figure 3. Overexpression of SHP2 enhances migration and invasion of MDA-MB-231 cells. (A) Wound-healing assay was performed to detect the cell migration. Width of the scratch showed the migration ability of the tumor cells. (B) Crystal violet staining of the membrane after Boyden chamber migration assay. (C) Invasive cells were counted using high-power field images at a magnification of $\mathrm{x} 200\left({ }^{* * *} \mathrm{P}<0.01\right)$.

\section{A}

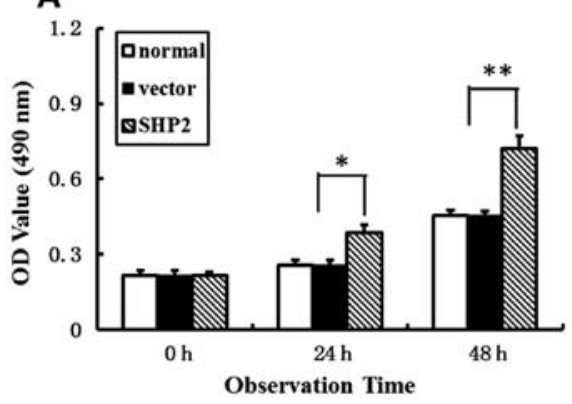

B

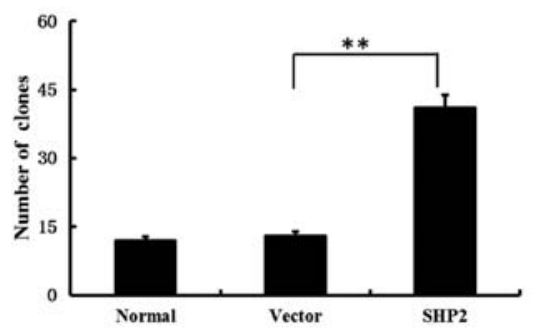

C

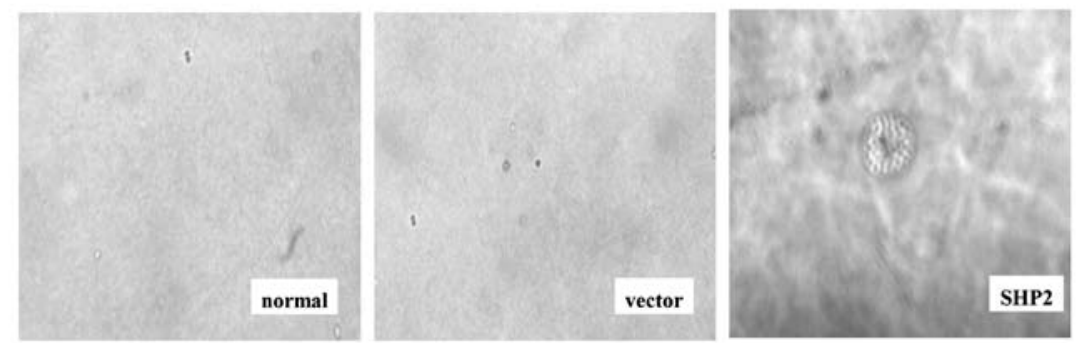

D

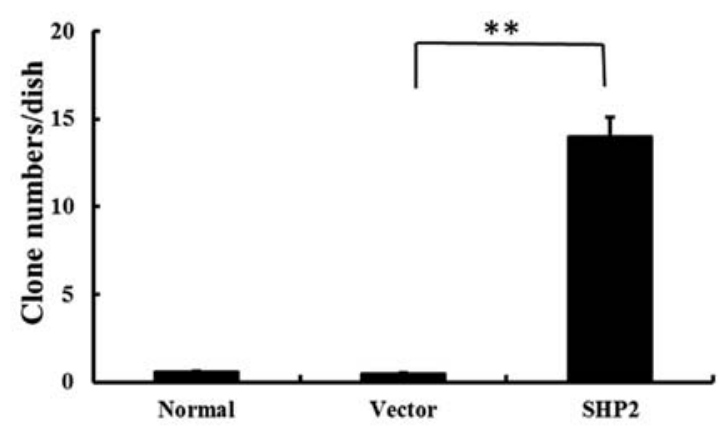

Figure 4. Overexpression of SHP2 accelerates the proliferation of MDA-MB-231 cells. (A) The growth ability of the MB-231 cells. (B) Foci were stained and counted based on triplicate wells for each cell line. (C) Anchorage-independent growth was observed by colony growth in soft agar. Representative microphotographs. Colonies formed by the SHP2-expressing cells are larger in shape. Representative images from experimental groups were selected randomly. (D) The number of clones was counted in the experimental groups. Each experiment was repeated a minimum of three times. Data shown are representative from at least three separate experiments with similar results. Significant differences at the same time points are presented $(" \mathrm{P}<0.05$, $\left.{ }^{* *} \mathrm{P}<0.01\right)$. 
A
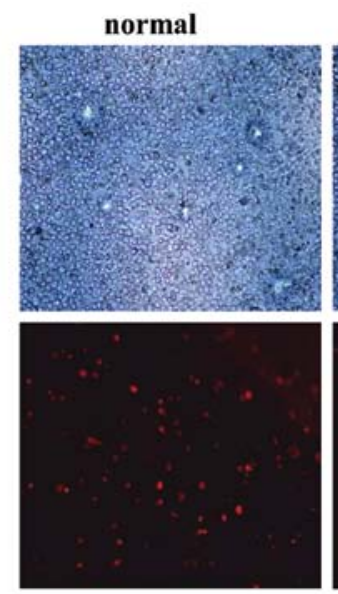

vector
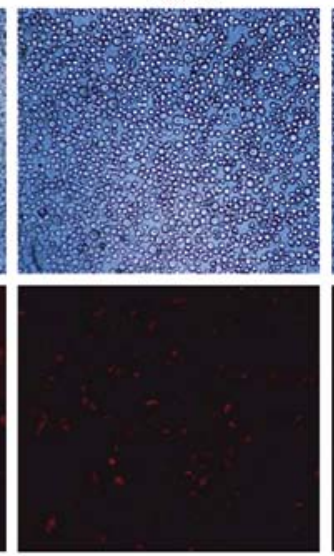

SHP2
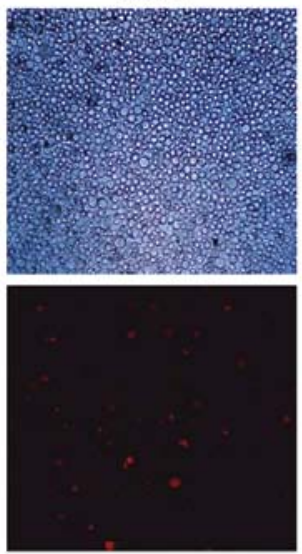

\section{B}

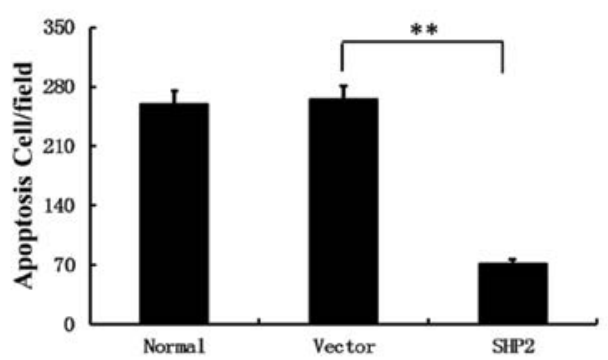

Figure 5. Overexpression of SHP2 is associated with increased resistance to chemotherapeutic agent cisplatin. (A) MTS assay was performed $24 \mathrm{~h}$ after incubation of MDA-MB-231 cells with cisplatin $(4 \mu \mathrm{g} / \mathrm{ml})$. (B) Apoptosis was analyzed after $24 \mathrm{~h}$ of incubation with $4 \mu \mathrm{g} / \mathrm{ml}$ cisplatin Absolute cell number was evaluated at this time point, and data are expressed as the percentage of growth compared to the vehicle control $\left({ }^{* *} \mathrm{P}<0.01\right)$.

Overexpression of SHP2 accelerates the proliferation of $M D A-M B-231$ cells. To determine whether the increase in cell migration, wound closure and invasion in breast cancer cells was due to increased cell growth, the proliferation was assessed by MTS assay. As shown in Fig. 4A, a significantly higher absorbance value at $490 \mathrm{~nm}$ was observed in the SHP2MB-231 group when compared with both control groups. These data suggest that the differences in actual cell migration were due to differential cell growth.

To further characterize the effect of SHP2 on MDA-MB231 cells, tumorigenesis was investigated. The focus formation experiment was performed, which reflected an increase in density-dependent or anchorage-independent growth in soft agar. The transfected MB-231 cells with SHP2 showed a significantly enhanced focus formation ratio. The number of clones in the SHP2-MB-231 group was markedly higher in comparison with the vector-transfected and non-transfected control groups (Fig. 4C and D). Anchorage-independent growth, as assessed by colony growth in soft agar, resulted in an up to 30-fold increase in the SHP2-MB-231 group than in control groups (Fig. 4D).

Overexpression of SHP2 increases resistance to chemotherapeutic agent cisplatin. To investigate the influence of SHP2 overexpression on cell proliferation, MST assay was performed after incubation of MDA-MB-231 cells with cisplatin. Cisplatin is a DNA-reactive agent which is commonly used in chemotherapy protocols for the treatment of breast cancer. As shown in Fig. 5A, in the control group, $4 \mu \mathrm{g} / \mathrm{ml}$ cisplatin inhibited the proliferation and increased the apoptosis of MB-231 cells in the control groups. In contrast, a significantly high proliferative rate and decreased apoptosis was observed in the SHP2-overexpressing MB-231 cells (Fig. 5A). In addition, fluorescence microscopy showed clear evidence of fragmented nuclei in the MDA-MB-231/empty vector control cells (Fig. 5A and B), which was markedly decreased in the SHP2-overexpression group. These data confirmed a positive role of SHP2 overexpression in MB-231 cell survival and proliferation.

Overexpressing of SHP2 promotes mammary tumor growth in mice. We demonstrated that the overexpression of SHP2 in MB-231 cells was associated with tumor invasion, proliferation and clone formation in an in vitro experiment. We next aimed to test whether SHP2 promotes the growth of MB-231 cells in an in vivo experiment. Therefore, a tumor xenograft model was established by subcutaneously implantation of MB-231 cells. Mammary tumors were detected 10 days after implantation (Fig. 6) in the vector-MB-231 and SHP2-MB-231 groups. The tumor size in the SHP2-MB-231 group continuously increased until day 50 after implantation (harvest point). In contrast, the tumor size in the vector-MB-231 control group remained at similar levels as day 10 until the end of the observation period. As shown in Fig. 6B, the size of solid tumors was significantly higher in the SHP2-MB-231 group than in the vector-MB231 control group. Moreover, the tumor weight in the SHP2MB-231 group was 20-fold higher when compared with the vector-MB-231 control group $(\mathrm{P}<0.01)$ (Fig. 6C). 
A
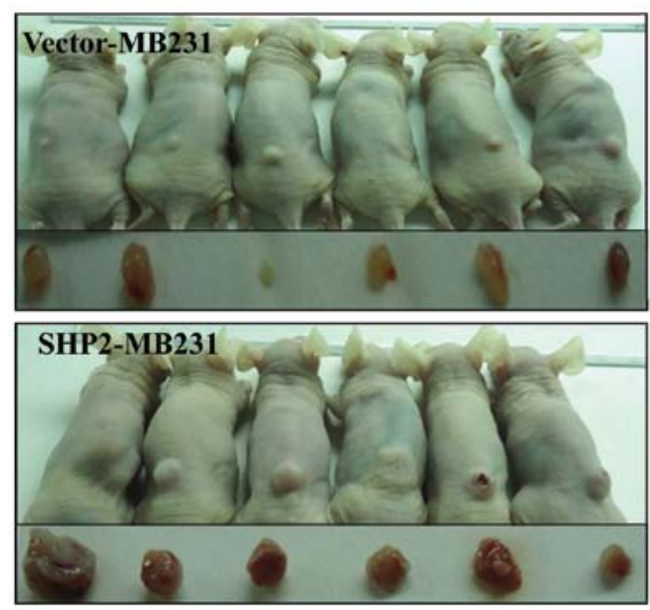

B
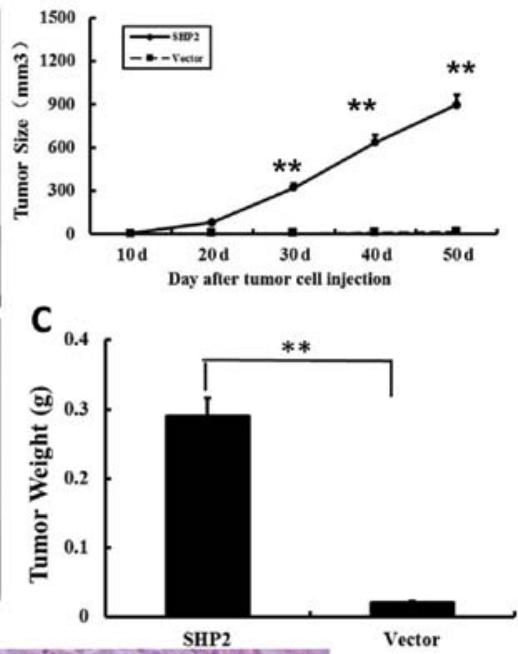

D
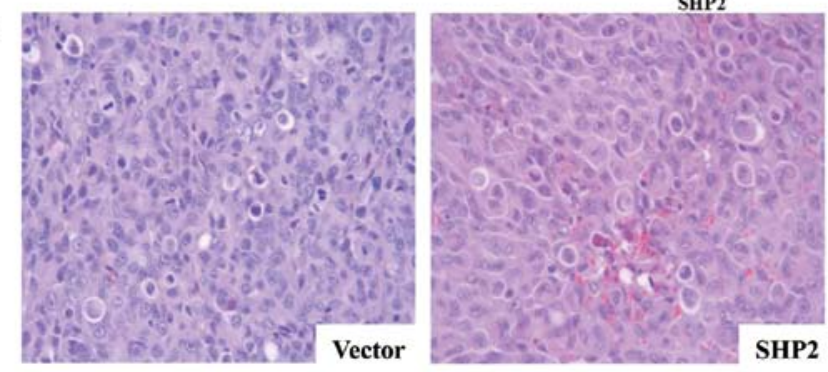

$\mathbf{E}$

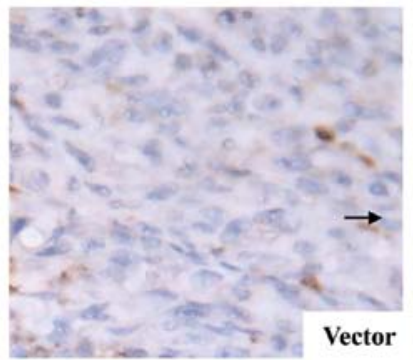

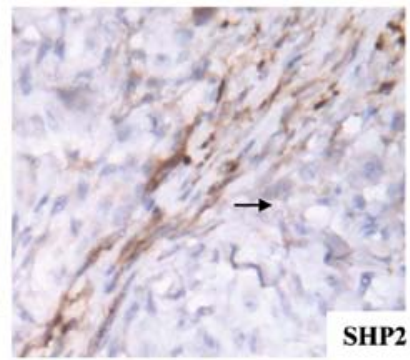

Figure 6. Overexpression of SHP2 promotes tumor growth and enhances tumor angiogenesis in a mouse xenograft model. (A) Representative image showing mammary tumors from the vector- (upper panel) and SHP2- (lower panel) MB-231 groups. Images were captured at the time point of sacrifice. (B) Tumor size was monitored every 10 days after implantation. (C) Solid tumors were removed and weighed after sacrifice. Data are shown as the means \pm SEM ( $\mathrm{n}=6 / \mathrm{group}$ ) $\left({ }^{* *} \mathrm{P}<0.01\right)$. (D) Morphological evaluation was assessed by performing H\&E staining. (E) CD31 IHC staining was performed to indicate angiogenesis in the solid tumor tissues. At least six areas from each group were examined.

SHP2 overexpression enhances tumor angiogenesis in mice. Since tumorigenesis is dependent on the sustained formation of new vessels, we determined the effect of SHP2 overexpression on tumor angiogenesis in the tumor xenograft model by performing CD31 IHC staining. As shown in Fig. 6E, there was an approximately multiple increase in AMVD in the tumor tissues from the SHP2-MB-231 group when compared with the tissue sections from the vector-MB-231 control groups. Similar observation was confirmed from morphological evaluations. These results suggest that SHP2 overexpression enhances tumor growth by promoting angiogenesis.

Overexpression of SHP2 contributes to elevated phosphoErk/AKT activation in breast cancer cells. SHP2 is a positive mediator of ligand-stimulated Ras activation. Previous studies have demonstrated that the positive effect of SHP2 on Ras activation is at least partially via the promotion of the phosphorylation of downstream ligand $(8,22,23)$. SHP2 is required in signal transduction to downstream MEK/ERK and PI-3K/AKT pathways $(8,23)$. Therefore, we examined the activation of MEK/ERK and PI-3K/AKT dependent kinase following SHP2 overexpression. Western blotting was performed to examine phosphorylated Erk and AKT, and their counterparts, respectively. In the SHP2-MB-231 cells, SHP2 overexpression resulted in significantly increased AKT phosphorylation (Fig. 7A and B). In addition, stimulation of MB-231 cells with EGF caused phosphorylation of ERK 5 and 15 min after incubation, respectively (Fig. 7C and D). Taken together, these data suggest that SHP2 regulates its downstream MEK/ ERK and PI-3K/Akt signaling pathways in a distinct manner in these cell lines and therefore may perturb cell cycle progression at different check-points.

\section{Discussion}

Upregulation of SHP2 has been widely observed in human breast cancer. The present study was designed to investigate the effect of SHP2 on the malignant phenotype of human breast 
A

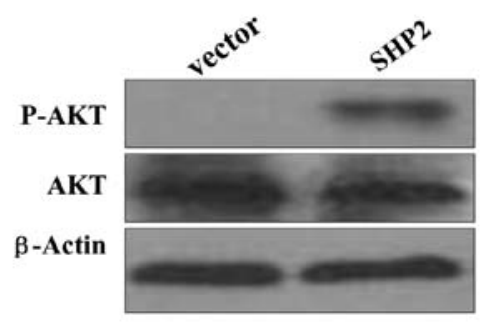

B

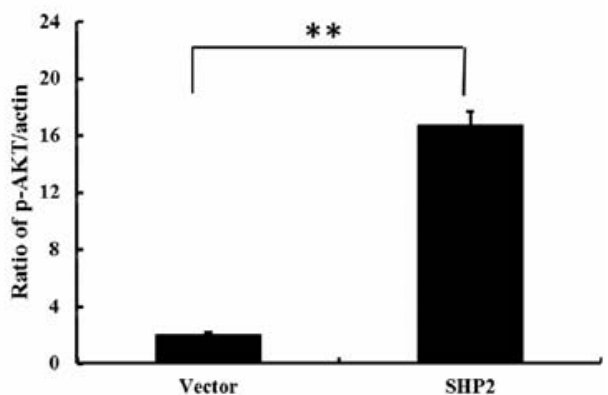

D

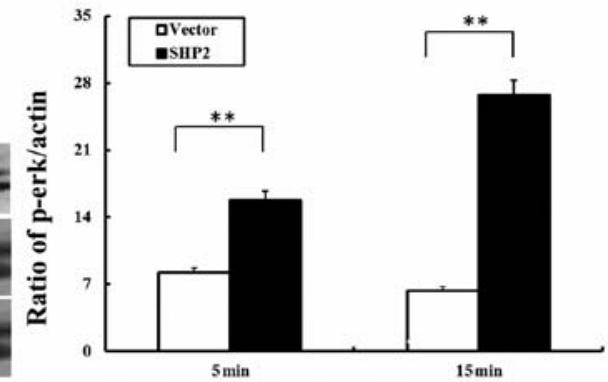

Figure 7. SHP2 overexpression contributes to elevated phospho-Erk/AKT activation in breast cancer cells. (A) Cells were starved for $24 \mathrm{~h}$ and stimulated in DMEM with $10 \%$ FBS for $2 \mathrm{~h}$. P-AKT, AKT and $\beta$-actin were detected by western blotting. (B) Densitometry of the bands from the western blot analysis was analyzed by Image J program. Quantitative analysis of P-AKT was normalized to $\beta$-actin protein levels. (C) MB-231 cells were starved overnight and incubated for 5 and $15 \mathrm{~min}$ with EGF $(5 \mathrm{ng} / \mathrm{ml})$. The activated form of P-ERK was detected by western blotting. (D) Densitometry of the bands from the western blot analysis was analyzed by Image $\mathrm{J}$ program. Quantitative analysis of P-ERK was normalized to $\beta$-actin protein levels $\left({ }^{* *} \mathrm{P}<0.01\right)$.

cancer. Our results indicated that overexpression of SHP2 was associated with increased cell proliferation, clone formation and decreased chemotherapeutic sensitivity. Transfection of SHP2 into breast cancer cells significantly promoted the tumor growth in a mouse xenograft model. Moreover, the mechanism of the promotion of tumorigenesis by SHP2 appears to involve its ability to increase the activity of ERK/AKT-mediated signaling pathways.

Upregulation of SHP2 expression is observed in advanced tumors $(9,20,24,25)$, and is accompanied by increased intracellular adhesion, and is essential for tumor metastasis (18). Therefore, overexpression of SHP2 in cancer has been considered as a mechanism mediating tumor metastasis (11-14). Our results indicate that upregulation of SHP2 expression in breast cancer cells promotes tumor invasiveness. Inhibition of SHP2 in breast cancer cells was found to cause transition of mesenchymal cells to epithelial cells, leading to decreased anchorage-independent cell growth (19). These observations suggest that SHP2 may play a central role in tumor malignancy. We found that the capabilities of adhesion and extension on fibronectin were significantly promoted in breast cancer cells following SHP2 transfection. This observation supports the hypothesis that overexpression of SHP2 accelerates the malignant potential of these cells. Formation of large, invasive breast tumors in mice after injection of the SHP2-MB-231 human breast tumor cells clearly demonstrates a critical role of SHP2 in the rapid development of breast tumors after tumor cell implantation.

Tumor angiogenesis is a crucial event in the growth of solid tumors. In the present study, angiogenesis in the solid tumors was increased after tumor cell implantation in the SHP2 overexpression group. Studies have demonstrated that a phenotype of tumor angiogenesis is switched on from the very early stages of tumor progression, in order to supply nutrients and oxygen. One marker of new blood vessel formation is CD31, a member of the immunoglobulin superfamily. Microvessel density can be quantitated as a representation of active tumor-associated angiogenesis by performing CD31 staining. Typically, microvessels are localized at the peripheral region of the solid tumor (26), which is consistent with our findings. In the present study, mass necrosis was observed in the central part of the solid tumors derived from the injection of MB-231 cells. A previous study showed a correlation between hypoxia and necrosis of solid tumors (27), revealing that the oxygen supply as well as angiogenesis might be important factors in tumor growth. Of note, no necrosis was observed in the solid tumors induced by implantation of the SHP2-overexpressing MB-231 cells. Moreover, significantly increased CD31 expression was observed as well in the same group. Our observations suggest that the angiogenic phenotype of the SHP2-overexpressing human tumor xenografts provides a survival advantage to the interior tumor epithelial cells.

Apoptosis is an essential factor to maintain homeostasis, and loss of apoptosis is an important characteristic of oncogenesis (17). Research indicates that SHP2 overexpression is associated with decreased apoptosis of tumor cells in a $3 \mathrm{D}$ culture model $(21,22)$. Our results indicated that MB-231 cells with SHP2 overexpression showed a significantly lower apoptotic rate following treatment with cisplatin. The transfection of SHP2 resulted in decreased cell death in the MB-231 cells and induced colony-formation ability in either 2D or 3D culture, suggesting that overexpression of SHP2 may be a causative factor in tumorigenesis.

Noteworthy, downregulation of most members of the $\mathrm{Sr}$ family is observed in tumors (8). This discrepancy may be due to tissue-specific molecular mechanisms (28). The function 
of SHP2 in the development of breast cancer needs further investigation.

SHP2 is required in signal transduction to downstream MEK/ERK and PI-3K/AKT pathways $(4,8,29)$. It is reported, that SHP2 interferes with MAPK cellular signal transduction via Erk, JNK/SAPK, p38/RK and BMK1/Erk5 in leukemic cell and in breast cancer MCF-7 cells (17). In the present study, we examined the activation of MEK/ERK and PI-3K/ AKT dependent kinase following SHP2 activation. We found that overexpression of SHP2 was associated with the activation of these signaling pathways in MB-231 cells. Therefore, overexpression of SHP2 may be necessary for mediating mammary tumorigenesis through promotion of Erk signaling or selective activation of PI3K signaling. These results reveal a critical role of SHP2 in breast cancer.

Taken together, our results indicate that overexpression of SHP2 in breast cancer cells leads to malignant transformation, and SHP2 appears to be a novel candidate for establishing new therapeutic strategies against breast cancer. Our results suggest that SHP2 may represent a key molecule in tumorigenesis, and blockade of SHP2 may be an effective immunotherapeutic strategy.

\section{Acknowledgements}

This study was supported by a grant from the National Natural Science Foundation of China (codes: 30873046, 30973424, 81072663 and 81272258 ) and by the Fundamental Research Funds for the Central Universities (2242014K40004).

\section{References}

1. Bentires-Alj M, Paez JG, David FS, et al: Activating mutations of the Noonan syndrome-associated SHP2/PTPN11 gene in human solid tumors and adult acute myelogenous leukemia. Cancer Res 64: 8816-8820, 2004

2. Ben-Jonathan N, Liby K, McFarland M and Zinger M: Prolactin as an autocrine/paracrine growth factor in human cancer. Trends Endocrinol Metab 13: 245-250, 2002.

3. Chan G, Kalaitzidis D and Neel BG: The tyrosine phosphatase Shp2 (PTPN11) in cancer. Cancer Metastasis Rev 27: 179-192, 2008.

4. Martinelli S, Carta C, Flex E, et al: Activating PTPN11 mutations play a minor role in pediatric and adult solid tumors. Cancer Genet Cytogenet 166: 124-129, 2006.

5. Miyamoto D, Miyamoto M, Takahashi A, Yomogita Y, Higashi H, Kondo S and Hatakeyama M: Isolation of a distinct class of gainof-function SHP-2 mutants with oncogenic RAS-like transforming activity from solid tumors. Oncogene 27: 3508-3515, 2008.

6. Grossmann KS, Rosario M, Birchmeier C and Birchmeier W: The tyrosine phosphatase Shp2 in development and cancer. Adv Cancer Res 106: 53-89, 2010.

7. Chan RJ and Feng GS: PTPN11 is the first identified protooncogene that encodes a tyrosine phosphatase. Blood 109: 862-867, 2007.

8. Serra V, Scaltriti M, Prudkin L, et al: PI3K inhibition results in enhanced HER signaling and acquired ERK dependency in HER2-overexpressing breast cancer. Oncogene 30: 2547-2557, 2011.

9. Tartaglia M, Mehler EL, Goldberg R, et al: Mutations in PTPN11, encoding the protein tyrosine phosphatase SHP-2, cause Noonan syndrome. Nat Genet 29: 465-468, 2001.

10. Edouard T, Combier JP, Nedelec A, et al: Functional effects of PTPN11 (SHP2) mutations causing LEOPARD syndrome on epidermal growth factor-induced phosphoinositide 3-kinase/ AKT/glycogen synthase kinase 3beta signaling. Mol Cell Biol 30: 2498-2507, 2010.
11. Masunaga R, Kohno H, Dhar DK, et al: Cyclooxygenase-2 expression correlates with tumor neovascularization and prognosis in human colorectal carcinoma patients. Clin Cancer Res 6: 4064-4068, 2000.

12. Zhou X, Coad J, Ducatman B and Agazie YM: SHP2 is up-regulated in breast cancer cells and in infiltrating ductal carcinoma of the breast, implying its involvement in breast oncogenesis. Histopathology 53: 389-402, 2008.

13. Dong Q, Siminovitch KA, Fialkow L, Fukushima T and Downey GP: Negative regulation of myeloid cell proliferation and function by the $\mathrm{SH} 2$ domain-containing tyrosine phosphatase-1. J Immunol 162: 3220-3230, 1999.

14. Agarwal R, D'Souza T and Morin PJ: Claudin-3 and claudin-4 expression in ovarian epithelial cells enhances invasion and is associated with increased matrix metalloproteinase-2 activity. Cancer Res 65: 7378-7385, 2005.

15. Frearson JA and Alexander DR: The phosphotyrosine phosphatase SHP-2 participates in a multimeric signaling complex and regulates T cell receptor (TCR) coupling to the Ras/mitogenactivated protein kinase (MAPK) pathway in Jurkat T cells. $\mathrm{J}$ Exp Med 187: 1417-1426, 1998.

16. Hadari YR, Kouhara H, Lax I and Schlessinger J: Binding of Shp2 tyrosine phosphatase to FRS2 is essential for fibroblast growth factor-induced PC12 cell differentiation. Mol Cell Biol 18: 3966-3973, 1998.

17. Carver KC, Piazza TM and Schuler LA: Prolactin enhances insulin-like growth factor I receptor phosphorylation by decreasing its association with the tyrosine phosphatase SHP-2 in MCF-7 breast cancer cells. J Biol Chem 285: 8003-8012, 2010.

18. Zhou XD and Agazie YM: Inhibition of SHP2 leads to mesenchymal to epithelial transition in breast cancer cells. Cell Death Differ 15: 988-996, 2008.

19. Zhou X and Agazie YM: Molecular mechanism for SHP2 in promoting HER2-induced signaling and transformation. J Biol Chem 284: 12226-12234, 2009.

20. Tartaglia M, Niemeyer CM, Fragale A, et al: Somatic mutations in PTPN11 in juvenile myelomonocytic leukemia, myelodysplastic syndromes and acute myeloid leukemia. Nat Genet 34: 148-150, 2003.

21. Eklund EA, Jalava A and Kakar R: PU.1, interferon regulatory factor 1 , and interferon consensus sequence-binding protein cooperate to increase gp91(phox) expression. J Biol Chem 273: 13957-13965, 1998.

22. Bouyain $S$ and Watkins DJ: Identification of tyrosine phosphatase ligands for contactin cell adhesion molecules. Commun Integr Biol 3: 284-286, 2010.

23. Wang S, Yu WM, Zhang W, McCrae KR, Neel BG and Qu CK: Noonan syndrome/leukemia-associated gain-of-function mutations in SHP-2 phosphatase (PTPN11) enhance cell migration and angiogenesis. J Biol Chem 284: 913-920, 2009

24. Tartaglia M, Kalidas K, Shaw A, et al: PTPN11 mutations in Noonan syndrome: molecular spectrum, genotype-phenotype correlation, and phenotypic heterogeneity. Am J Hum Genet 70: 1555-1563, 2002.

25. Tartaglia M, Martinelli S, Iavarone I, et al: Somatic PTPN11 mutations in childhood acute myeloid leukaemia. Br J Haematol 129: 333-339, 2005.

26. Chen H, Pimienta G, Gu Y, et al: Proteomic characterization of Her2/neu-overexpressing breast cancer cells. Proteomics 10: 3800-3810, 2010.

27. Nijsten T, Colpaert CG, Vermeulen PB, Harris AL, Van ME and Lambert J: Cyclooxygenase-2 expression and angiogenesis in squamous cell carcinoma of the skin and its precursors: a paired immunohistochemical study of 35 cases. Br J Dermatol 151: 837-845, 2004

28. Van IC, Rahner C and Anderson JM: Regulated expression of claudin-4 decreases paracellular conductance through a selective decrease in sodium permeability. J Clin Invest 107: 1319-1327, 2001.

29. Montagner A, Yart A, Dance M, Perret B, Salles JP and Raynal P: A novel role for Gab1 and SHP2 in epidermal growth factorinduced Ras activation. J Biol Chem 280: 5350-5360, 2005. 